\title{
Direct Visualization of Solution-based Nanofabrication Processes with In Situ TEM: Chemical Wet-etching and Solution-based Cleaning/Drying of High-Aspect-Ratio Nanostructures.
}

Utkur Mirsaidov $^{1,2,3}$, Zainul Aabdin ${ }^{1,4}$, Tanmay Ghosh ${ }^{1,2}$, Utkarsh Anand ${ }^{1,2}$, Nandi Vrancken ${ }^{5}$, Xiu Mei Xu ${ }^{5}$, See Wee Chee ${ }^{1,2}$, Zhaslan Baraissov ${ }^{1,2}$, and Frank Holsteyns ${ }^{5}$

1. Center for Advanced 2D Materials and Department of Physics, National University of Singapore, Singapore 117546,

2. Center for BioImaging Sciences and Department of Biological Sciences, National University of Singapore, Singapore 117557,

3. Department of Materials Science and Engineering, National University of Singapore, Singapore 117575 ,

4. Institute of Materials Research and Engineering, A*STAR (Agency for Science, Technology and Research), Singapore 138634, Singapore,

5. imec, Kapeldreef 75, Leuven, B-3001, Belgium.

Controlled fabrication of 3D nanoscale materials from semiconductors is important for many technologies. For example, scaling up the density of the transistors per chip requires the fabrication of smaller and smaller vertical nanowires as channel materials [1]. Two key processes essential to the fabrication of these devices is precise etching of the nanostructures and the damage-free solution based cleaning (damage occurs during post-clean drying due to capillary forces). However, very little is known about both of these processes because it is extremely challenging to visualize etching and cleaning with solutions directly at the nanoscale. Here, using in situ liquid phase dynamic TEM imaging [2-4], we first describe the detailed mechanisms of etching of vertical Si nanopillars in alkaline solutions [5]. Our design of liquid cells includes a periodic array of patterned nanopillars at a density of $1.2 \times 10^{10} \mathrm{~cm}^{-2}$ (Figure 1A). We show that the nanoscale chemical wet-etch of Si occurs in three stages as schematically shown in Figure 1B: 1) intermediates generated during alkaline wet etching aggregate as nanoclusters on the Si surface, 2) then these intermediates detach from the surface before 3) dissolving in the etchant solution.

Next, we describe the capillary damage of these high-aspect-ratio Si nanopillars during drying after the solution-phase cleaning. Our results reveal that drying induced damage to nanopillars occurs in three distinct steps. First, as water evaporates from the surface patterned with nanopillars, water film thin down non-uniformly leaving small water nanodroplets trapped between the nanopillars. Second, the capillary forces induced by these droplets bend and bring the nanopillars into contact with each other at which point they bond together. Third, droplets trapped between the nanopillars evaporate leaving the nanopillars bonded to each other. We show that even after the nanodroplets finally evaporate, interfacial water covering the nanopillars act as a glue and holds the pillars together.

Our findings highlight the importance of being able to visualize the processes relevant to nanofabrication in order to resolve the failure modes that will occur more frequently as the device sizes get even smaller in future. 


\section{References:}

[1] C. Thelander et al, Mater. Today 9 (2006), 28-35.

[2] M. J. Williamson et al, Nature Materials 2 (2003), p. 532.

[3] H. Zheng et al, Science 324 (2009), p. 1309.

[4] U. Mirsaidov et al, Proc. Natl. Acad. Sci. U.S.A. 109 (2012), p. 7187.

[5] Z. Aabdin et al, Nano Letters 17 (2017), p.2953.

[6] This work was supported by Singapore National Research (NRF-CRP16-2015-05).

A

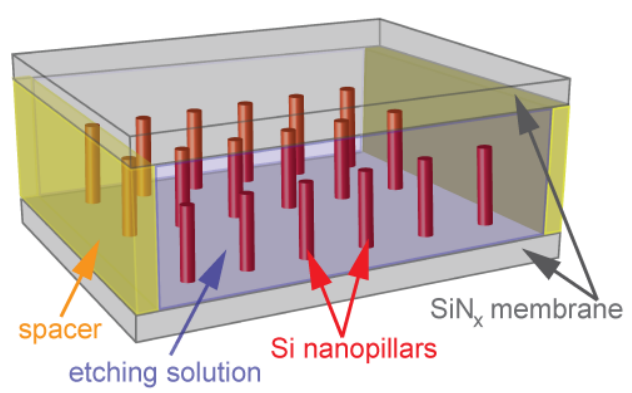

B etch intermediates form nanoclusters on the surface nanoclusters grow nanoclusters detach on the surface and dissolve

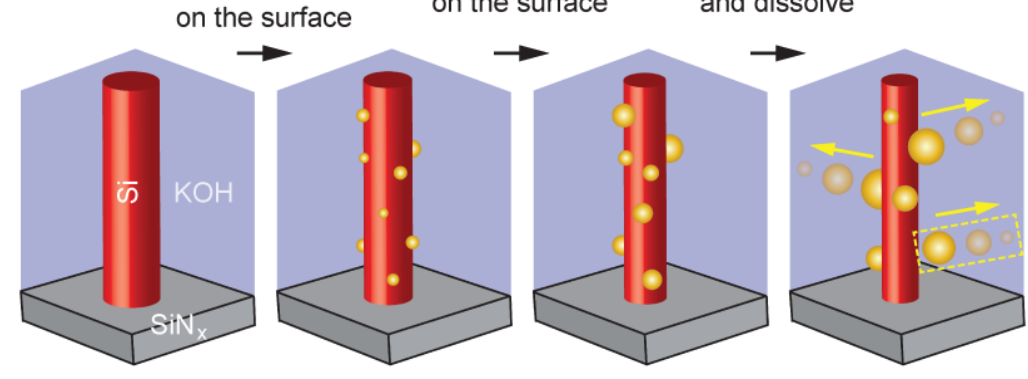

Figure 1. (A) Schematic of our liquid cell platform with Si nanopillars used for in situ TEM imaging of wet etching process. An array of $200 \mathrm{~nm}$ tall and $45 \mathrm{~nm}$ diameter Si nanopillars are patterned on a bottom $\mathrm{SiN}_{\mathrm{x}}$ membrane at a pitch of $90 \mathrm{~nm}$; a 240-nm spacer separates the two membranes. The nanopillars are then immersed in an etchant $(\mathrm{KOH})$ solution that fills the liquid cell, and the resulting etching process is observed inside a TEM. (B) Schematic illustration of a two-step etching mechanism: (i) formation and growth of reaction intermediates on the surface of the nanopillars, followed by (ii) detachment and (iii) subsequent dissolution of these intermediates in the etchant solution. 